\title{
Cost-Utility Analysis of Mycophenolate Mofetil versus Azathioprine Based Regimens for Maintenance Therapy of Proliferative Lupus Nephritis
}

\author{
Robert Nee, Ian Rivera, Dustin J. Little, Christina M. Yuan, and Kevin C. Abbott \\ Department of Nephrology, Walter Reed National Military Medical Center, 8901 Wisconsin Avenue, Bethesda, MD 20889-5600, USA \\ Correspondence should be addressed to Robert Nee; robert.nee.civ@mail.mil
}

Received 13 July 2015; Revised 23 September 2015; Accepted 29 September 2015

Academic Editor: Kazunari Kaneko

Copyright (c) 2015 Robert Nee et al. This is an open access article distributed under the Creative Commons Attribution License, which permits unrestricted use, distribution, and reproduction in any medium, provided the original work is properly cited.

Background/Aims. We aimed to examine the cost-effectiveness of mycophenolate mofetil (MMF) and azathioprine (AZA) as maintenance therapy for patients with Class III and Class IV lupus nephritis (LN), from a United States (US) perspective. Methods. Using a Markov model, we conducted a cost-utility analysis from a societal perspective over a lifetime horizon. The modeled population comprised patients with proliferative LN who received maintenance therapy with MMF (2 gm/day) versus AZA ( $150 \mathrm{mg} /$ day) for 3 years. Risk estimates of clinical events were based on a Cochrane meta-analysis while costs and utilities were retrieved from other published sources. Outcome measures included costs, quality-adjusted life-years (QALY), incremental cost-effectiveness ratios (ICER), and net monetary benefit. Results. The base-case model showed that, compared with AZA strategy, the ICER for MMF was $\$ 2,630,592 /$ QALY at 3 years. Over the patients' lifetime, however, the ICER of MMF compared to AZA was $\$ 6,454 /$ QALY. Overall, the ICER results from various sensitivity and subgroup analyses did not alter the conclusions of the model simulation. Conclusions. In the short term, an AZA-based regimen confers greater value than MMF for the maintenance therapy of proliferative LN. From a lifelong perspective, however, MMF is cost-effective compared to AZA.

\section{Introduction}

Lupus nephritis (LN) is a serious and costly cause of kidney disease worldwide [1]. An analysis of United States (US) medical expenditures found that the annual costs per patient among those with LN exceeded $\$ 46,000$ (USD) versus matched controls and $\$ 42,000$ versus systemic lupus erythematosus (SLE) patients without nephritis [2]. These findings suggest that LN is a key driver of economic burden in the SLE population.

The Kidney Disease Improving Global Outcomes (KDIGO) practice guidelines for initial or induction therapy for LN are well accepted [3]; however, they do not indicate a preference for maintenance therapy with azathioprine(AZA-) or mycophenolate mofetil- (MMF-) based regimens. The Task Force Panel of the American College of Rheumatology recommended that either AZA or MMF be used for maintenance [4]. These recommendations were based, in large part, on two randomized controlled trials of long-term maintenance therapies for LN. In the MAINTAIN Nephritis Trial, a predominantly Caucasian cohort was randomized to MMF $2 \mathrm{gm} /$ day or AZA $2 \mathrm{mg} / \mathrm{kg} /$ day as maintenance therapy after induction with a fixed, low dose intravenous (IV) cyclophosphamide (CYC) regimen [5]. After a mean followup of 4 years, this European-based study found that MMF was not superior to AZA in preventing renal flares, without significant differences in adverse events except for higher rate of cytopenias in the AZA group. In the larger Aspreva Lupus Management Study (ALMS) trial, a multinational population was randomized to MMF $2 \mathrm{gm} /$ day or AZA $2 \mathrm{mg} / \mathrm{kg} / \mathrm{day}$ after response to initial induction therapy [6]. After 3 years, MMF was superior to AZA as maintenance therapy, based on the primary composite end point of death, end stage renal disease (ESRD), doubling of the serum creatinine, renal flare, or requirement for rescue therapy.

To our knowledge, a cost-effectiveness analysis of maintenance therapy for proliferative LN from a US perspective has not been reported. We conducted a cost-utility analysis 


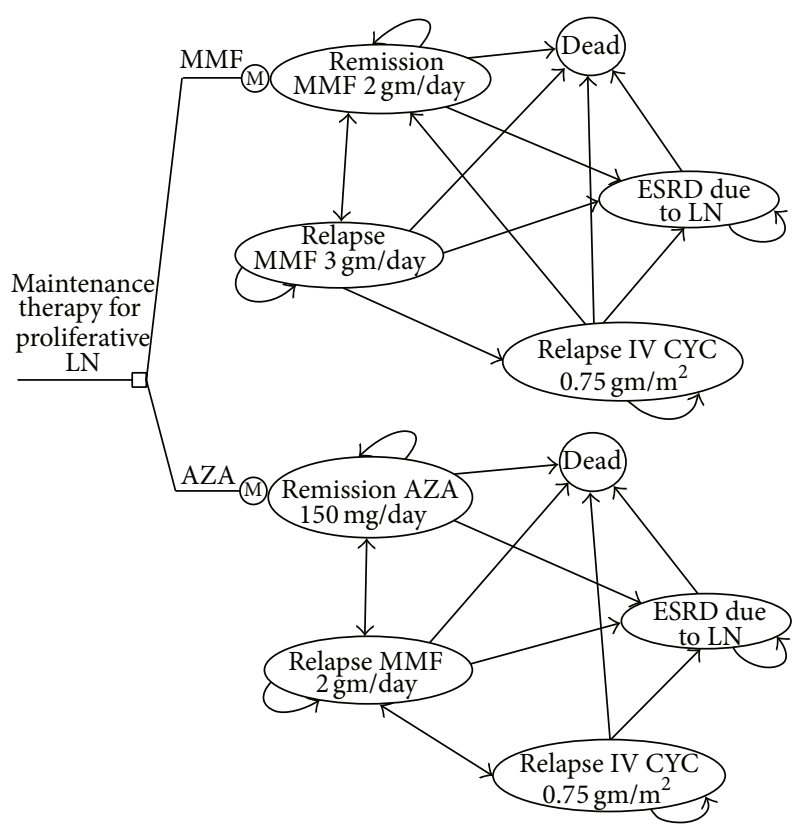

(a)

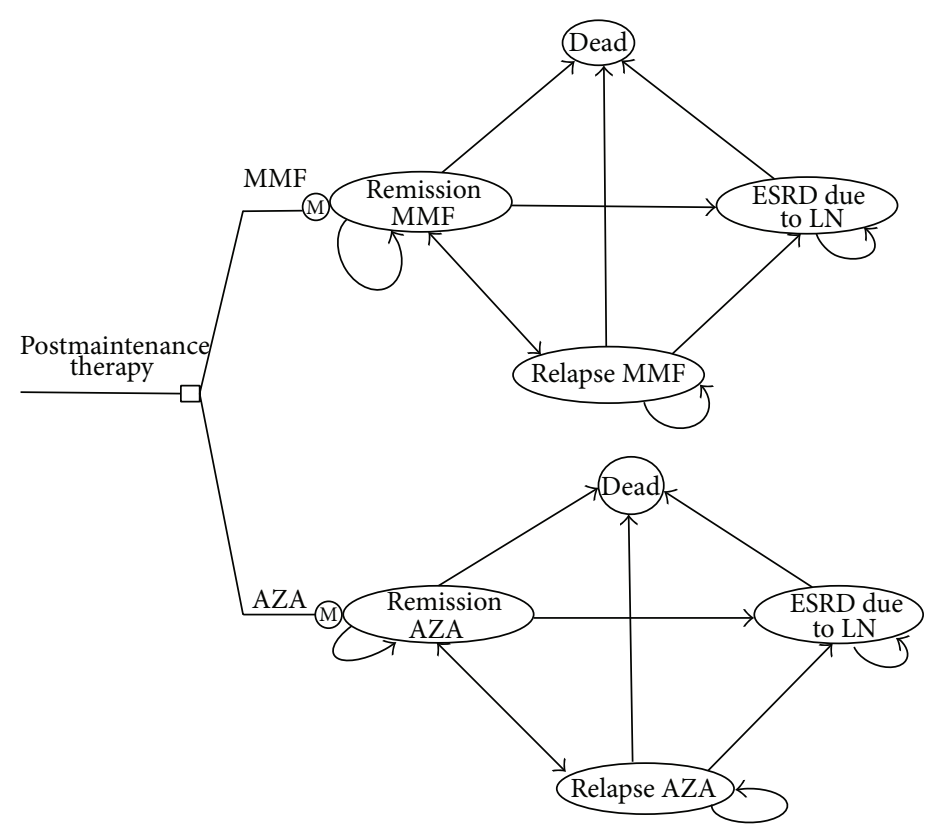

(b)

FIGURE 1: (a) Markov state transition diagram illustrating the health states and transitions for each treatment strategy for the initial 3 years. The lifetime model consists of the initial 3-year period of maintenance therapy followed by a posttreatment phase as shown in (b). (b) Markov state transition diagram illustrating the health states and transitions for each treatment strategy for the posttreatment phase (after 3 years). LN: lupus nephritis; AZA: azathioprine; MMF: mycophenolate mofetil; IV CYC: intravenous cyclophosphamide; ESRD: end stage renal disease.

from a societal perspective to evaluate the cost-effectiveness of the 3-year maintenance regimens (MMF versus AZA) for proliferative LN over a lifetime horizon.

\section{Methods}

2.1. Study Design. We constructed a Markov state transition model to estimate the quality-adjusted life-years (QALY) and costs associated with maintenance therapy with MMF versus AZA. Markov models analyze uncertain events over time and are suited to decisions where the timing of events is important and when events are recursive in nature [7]. While decision trees model uncertain events at chance nodes, Markov models analyze these events as transitions between health states. Markov models are suited to modelling chronic conditions, where costs and outcomes (QALY) are spread over a long period of time.

Our model encompasses an initial 3-year treatment phase after which simulated patients are no longer on immunosuppressive agents and followed long term (Tables 1(a) and 2(a); Supplemental Data Sources) (see Supplementary Material available online at http://dx.doi.org/10.1155/2015/917567). The time horizon in Markov models is divided into discrete time periods, called cycles. Each cycle length in our model is 6 months for the first 3 years and 12 months thereafter, reflecting the natural history of renal flares and remissions in LN. Analyses were performed using TreeAge Pro 2012 (TreeAge Software, Williamstown, MA) and Microsoft Excel 2010 (Microsoft Corp., Redmond, WA). Institutional review board approval was not required for this study. We adhered to the CHEERS (Consolidated Health Economic Evaluation Reporting Standards) reporting guidelines in our study [8].

2.2. Target Population. The modeled population is a hypothetical cohort of 1,000 patients with proliferative $\mathrm{LN}$ receiving maintenance therapy, having responded to their induction regimen. The starting age ranges from 20 to 40 years old, and various racial/ethnic groups are represented in the model, reflecting the demographic characteristics of study participants in the clinical trials.

2.3. Model Structure. The general structure of the model is shown as a state transition diagram in Figures 1(a) and 1(b) and Markov cycle trees (Supplemental Model Structure, Model Assumptions, and Supplemental Figures 1 and 2). We did not include the costs and QALY with induction therapy for proliferative LN given that this was a study of the differences between two maintenance treatment strategies and inclusion would not alter the conclusion of the analysis. We attempt to simulate patient-oriented outcomes and treatment strategies that are typically utilized in "real-world" clinical practice. For both strategies, after model entry each patient would progress through five potential health states, in 6month cycles:

(1) Remission state on MMF or AZA as maintenance therapy;

(2) relapse of $L N$ requiring MMF rescue therapy (escalation of MMF dose if maintained on MMF); 


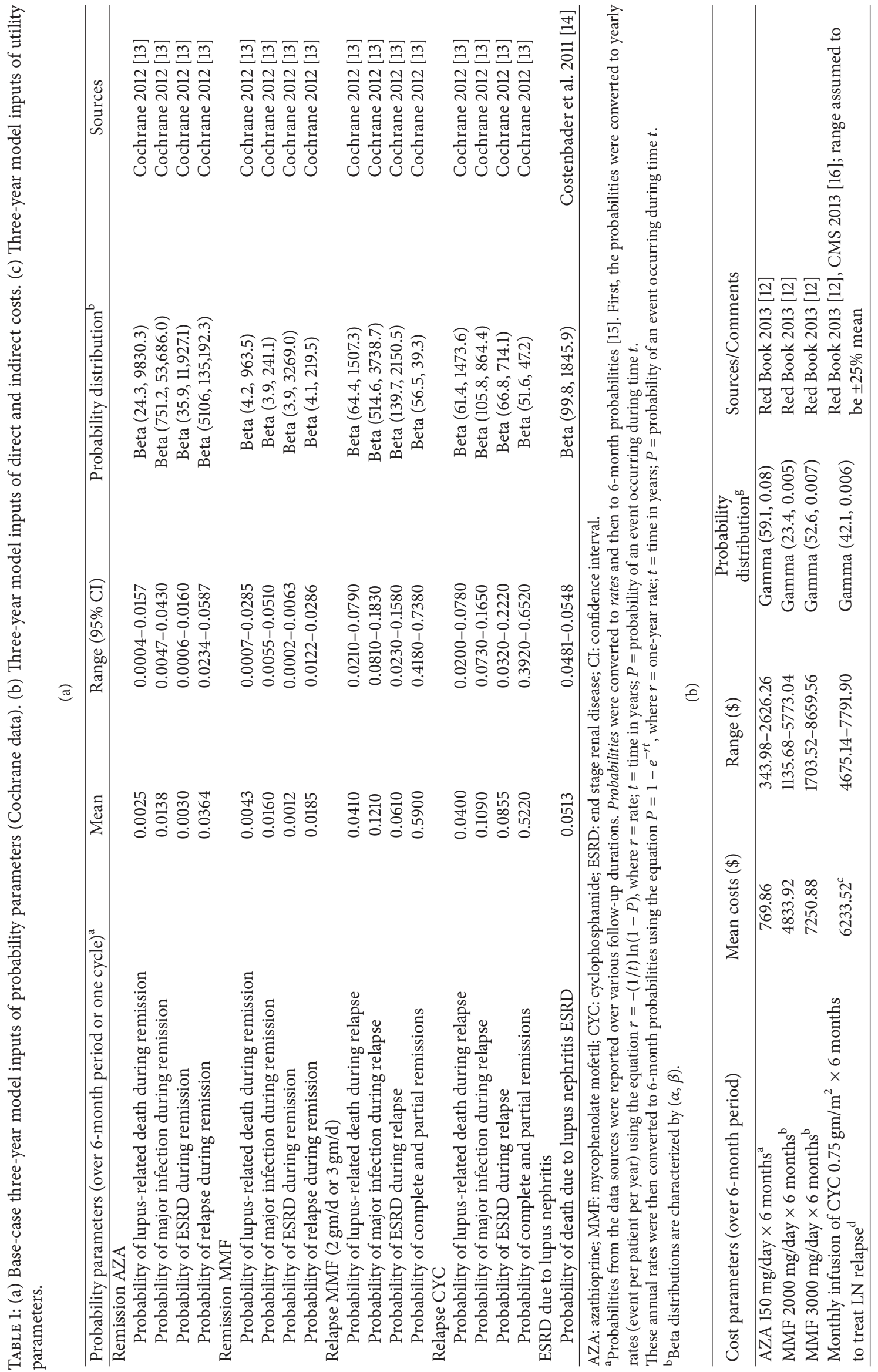




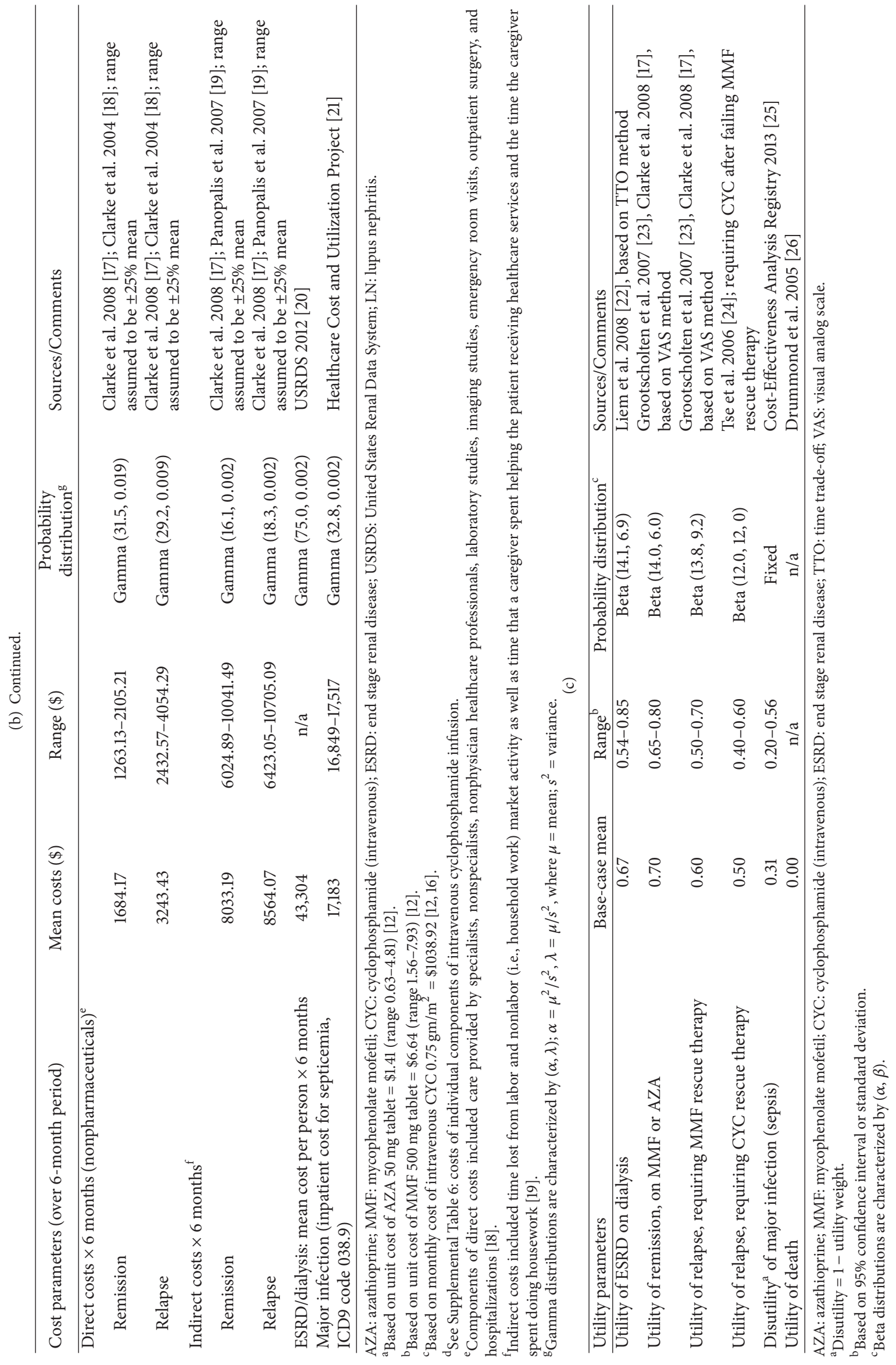




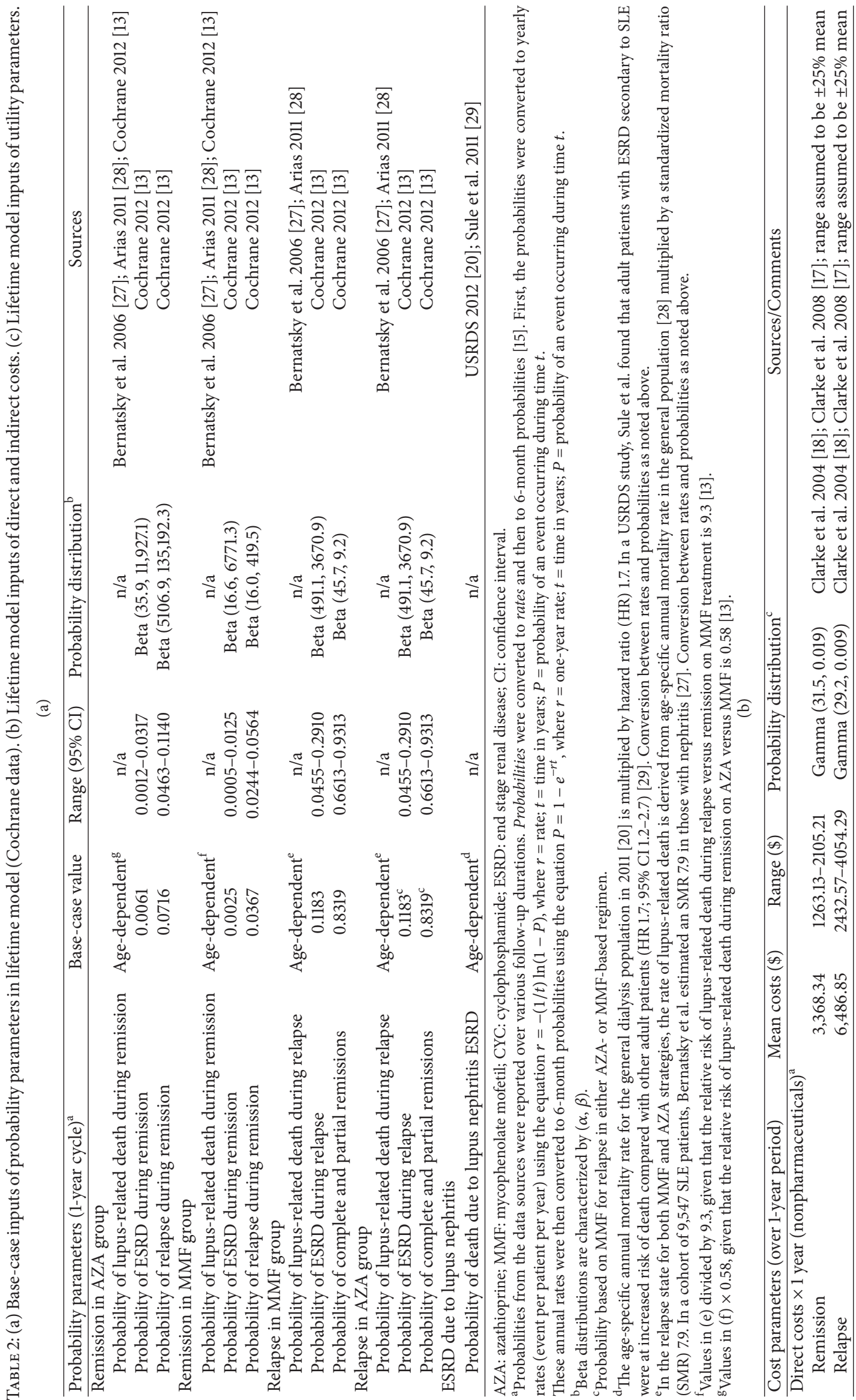




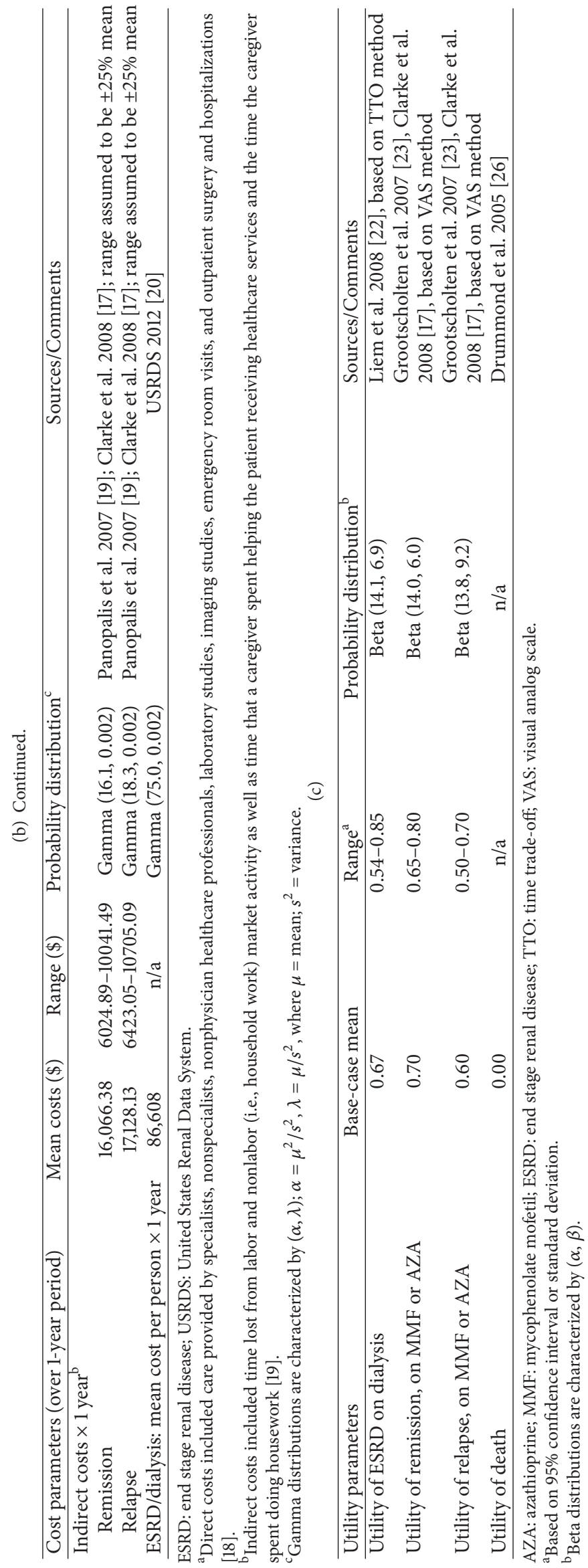


(3) relapse of LN despite MMF rescue therapy, requiring monotherapy with IV CYC;

(4) ESRD due to LN;

(5) death.

Upon completing the 3-year maintenance therapy, patients in each arm are assumed to be off the immunosuppressive medications and would progress through four potential health states in the lifetime model, in 12-month cycles:
(1) Remission;
(2) relapse of $\mathrm{LN}$;
(3) ESRD due to LN;
(4) death.

2.4. Interventions. We evaluated MMF (2 gm/day) and AZA $(150 \mathrm{mg} /$ day) as maintenance therapy for LN. The model accounted for sequential rescue therapy during 3 years of maintenance therapy. There is a paucity of clinical trial data on the treatment of LN flares. Therefore, the treatment approach in our model reflects the current recommendations of national and international experts $[9,10]$.

2.5. Costs. Costs of healthcare products and services were undertaken from a societal perspective. All costs were adjusted for inflation to 2013 US dollars by using the Consumer Price Index for Medical Care [11]. Drug costs are based on average wholesale prices (AWP) [12]; other cost items were obtained from previous literature and public sources. Tables 1(b) and 2(b) show the components of direct and indirect costs incurred during the 3-year maintenance therapy with either MMF or AZA and thereafter in the lifetime model (Supplemental Costs). As noted above, patients are assumed to be off immunosuppressive therapy after 3 years; therefore, costs of MMF, AZA, and CYC are not included in the lifetime model.

2.6. Utilities (QALY). QALY is the product of the utility score and the number of years spent in a particular health state. A utility score reflects preference of a surveyed sample of individuals for a particular health state; a preference score of 1.0 represents perfect health, whereas a 0 score represents death. Tables 1 (c) and 2(c) show the various utility weights of the health states in the model, obtained from previous literature (Supplemental Utilities).

2.7. Outcome Measures. The first outcome measure is the incremental cost-effectiveness ratios (ICER) which is the difference in costs between two strategies divided by the difference in effectiveness [7]:

$$
\operatorname{ICER}=\frac{\Delta C}{\Delta E}=\frac{\left(C_{1}-C_{2}\right)}{\left(E_{1}-E_{2}\right)},
$$

where $C_{1}$ is the cost of strategy $1, C_{2}$ is the cost of strategy 2 , $E_{1}$ is the QALY of strategy 1 , and $E_{2}$ is the QALY of strategy 2 .
The second outcome measure is the net monetary benefit (NMB) which represents the difference between the monetary value of an incremental QALY and the cost of achieving the benefit. The strategy with the highest NMB is the most cost-effective given a WTP parameter [7]

$$
\mathrm{NMB}=(E \times \lambda)-C,
$$

where $E$ is effectiveness (QALY), $\lambda$ is WTP, and $C$ is cost.

WTP is the amount society that is willing to pay for an additional QALY. We used a WTP of $\$ 50,000-\$ 100,000$ per QALY gained, often cited as the cost-effectiveness threshold in the literature [30].

2.8. Data Analysis. Our model is based on Reference Case analysis, a standard set of methodological practices for costeffectiveness analysis [31]. We conducted a two-dimensional simulation via a combination of probabilistic sensitivity analysis (PSA) and microsimulation [32] (Supplemental Data Analysis). We conducted sensitivity analyses to assess uncertainty in our model (Supplemental Sensitivity Analysis). We also conducted value of information analyses, using NMB calculations from the 3-year base-case model, to estimate the expected benefit of future research [32] (Supplemental Expected Value of Perfect Information). Total costs and QALY were calculated after six 1/2-year cycles in the 3-year model and after forty 1-year cycles in the base-case lifetime model.

\section{Results}

3.1. Model Validation. In assessing external validity, we compared predicted outputs from the 3-year model with observed data, which were generally comparable and within standard deviations (Supplemental Model Validation, Assessment of External Validity, and Supplemental Table 7). We also compared simulated 10-year and 15-year survival rates from the lifetime model with actual event data $[33,34]$. Overall, the predicted outcomes from the lifetime model approximated observed data from these studies (Supplemental Assessment of External Validity).

\subsection{3-Year Model}

\subsubsection{Base-Case Analysis (Cochrane Data)}

(i) Cost-Effectiveness. Compared with an AZA-based regimen, MMF had an incremental cost of $\$ 17,611$ and gain of 0.0067 QALY, with an ICER of $\$ 2,630,592$ per QALY (Table 3(a)).

(ii) Sensitivity Analyses. In a one-way sensitivity analysis, the MMF-based regimen was the favored strategy if the 6-month cost of MMF $2 \mathrm{gm} /$ day was $<\$ 954.13$ at WTP $\$ 50,000 /$ QALY (Supplemental Figure 3). This is equivalent to $\$ 1.33$ per $500 \mathrm{mg}$ MMF tablet and represents $20.0 \%$ of the actual AWP. As shown in Table 3(b), we conducted other sensitivity analyses by excluding indirect costs, varying utility weights or changing model assumptions; the ICER (MMF 
TABLE 3: (a) Costs, effectiveness, and incremental cost effectiveness ratios (ICER) of the base-case and individual clinical trials in three-year model. (b) Sensitivity analysis of three-year model using Cochrane data.

(a)

\begin{tabular}{|c|c|c|c|c|c|}
\hline Scenarios & Total cost (\$) & $\begin{array}{c}\text { Total } \\
\text { effectiveness } \\
\text { (QALY) }\end{array}$ & $\begin{array}{c}\text { Incremental } \\
\text { costs }(\$)\end{array}$ & $\begin{array}{c}\text { Incremental } \\
\text { effectiveness } \\
(\text { QALY })\end{array}$ & $\begin{array}{c}\text { ICER } \\
\text { (\$/QALY) }\end{array}$ \\
\hline \multicolumn{6}{|c|}{ Cochrane (base-case) } \\
\hline AZA & $54,249.98$ & 1.6367 & & & \\
\hline MMF & $71,861.21$ & 1.6434 & $17,611.23$ & 0.0067 & $2,630,591.76$ \\
\hline \multicolumn{6}{|l|}{ Subgroups } \\
\hline \multicolumn{6}{|l|}{ ALMS } \\
\hline AZA & $55,959.12$ & 1.6125 & & & \\
\hline MMF & $72,619.05$ & 1.6363 & $16,659.92$ & 0.0238 & $700,001.12$ \\
\hline \multicolumn{6}{|c|}{ MAINTAIN } \\
\hline AZA & $54,527.62$ & 1.6318 & & & \\
\hline MMF & $72,511.65$ & 1.6148 & $17,984.04$ & -0.0170 & Dominated \\
\hline
\end{tabular}

QALY: quality-adjusted life-years; AZA: azathioprine; MMF: mycophenolate mofetil.

(b)

\begin{tabular}{lc}
\hline Scenarios & ICER MMF versus AZA (US\$) \\
\hline Base-case & $2,630,591.76$ \\
Excludes indirect costs in both strategies & $2,529,609.93$ \\
Utility & $1,476,631.93$ \\
Remission = 0.8 (versus base-case 0.7) & $1,654,369.09$ \\
Relapse requiring MMF = 0.5 (versus base-case 0.6) & $2,555,137.00$ \\
Utility of relapse requiring CYC = utility of relapse requiring MMF rescue & $2,410,632.95$ \\
Conditions biased against AZA-based strategy & $1,380,997.67$ \\
Indirect costs $\times 6$ months during remission $(\$ 10,041.49)$ [higher indirect costs for AZA group] & \\
Indirect costs $\times 6$ months during remission $(\$ 10,041.49)+$ utility of remission state $(0.8)$ [higher & $709,870.18$ \\
indirect costs for AZA group + higher utility during remission] & \\
Indirect costs $\times 6$ months during remission $(\$ 10,041.49)+$ utility of remission state $(0.8)+$ drug & \\
costs of AZA $\times 6$ months $(\$ 2626)$ [higher indirect costs for AZA group + higher utility during & \\
remission + higher drug costs of AZA] & $1,900,694.28$ \\
Revised assumptions & $2,273,422.51$ \\
AZA group receives 3 gm/day of MMF as rescue (base-case 2 gm/day MMF) & \\
Patients in the AZA group who remit on CYC rescue therapy are treated with AZA maintenance \\
therapy (base-case MMF 2 gm/day)
\end{tabular}

ICER: incremental cost effectiveness ratio; AZA: azathioprine; MMF: mycophenolate mofetil.

versus AZA) of these analyses far exceeded the standard WTP \$50,000-\$100,000/QALY thresholds.

(iii) Tornado Analysis. At a WTP \$50,000/QALY, the model was most sensitive to (1) indirect costs during remission; (2) utility weight of the remission state; (3) drug price of AZA $150 \mathrm{mg} /$ day (Figure 2). These three parameters accounted for $82.4 \%$ of the total model uncertainty.

(iv) Scenario Analysis. Despite simulated conditions biased against AZA, the MMF-based regimen remained cost ineffective compared to its alternative at 3 years, with an ICER $\$ 709,870$ per QALY (Table 3(b)). (v) Probabilistic Sensitivity Analyses. The incremental costeffectiveness (ICE) scatterplot and the cost-effectiveness acceptability curve (CEAC) showed that an AZA-based regimen had a near $100 \%$ probability of being cost-effective over a 3-year time frame, at WTP thresholds of $\$ 50,000$ and $\$ 100,000 /$ QALY (Figure 3(a), Supplemental Figure 4).

(vi) Expected Value of Perfect Information (EVPI). The population EVPI represents the upper bound on the expected gain on investment on further data collection, which we calculated to be $\$ 2,058,206$ at WTP $\$ 100,000 /$ QALY in the US population, assuming a period of 10 years with $3 \%$ discount rate (Supplemental Expected Value of Perfect Information). 


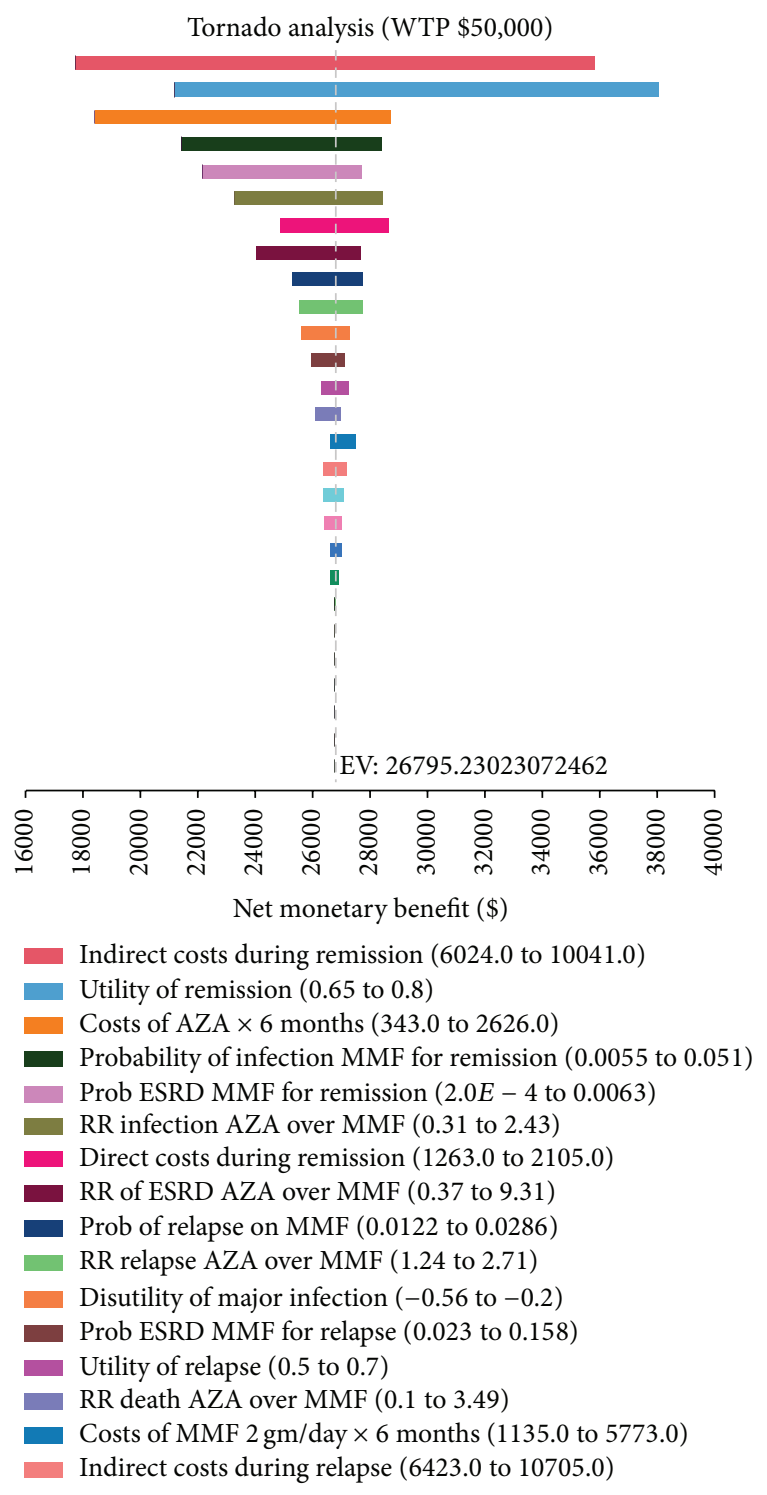

Figure 2: Tornado diagram of the 3-year base-case model, demonstrating one-way sensitivity analysis of each variable in the model. Each bar represents a range of expected values (EV), expressed as net monetary benefit in US dollars, over plausible estimates for an individual variable. The dotted vertical line indicates the base-case expected value. WTP: willingness-to-pay.

3.2.2. Subgroup Analysis. Based on ALMS data, MMF had an ICER of $\$ 700,001$ per QALY compared with an AZAbased regimen (Table 3(a)). Furthermore, AZA was both cost-saving and more effective than MMF using data from the MAINTAIN trial (Table 3(a)).

\subsection{Lifetime Model (40 Years)}

\subsubsection{Base-Case Analysis (Cochrane Data)}

(i) Cost-Effectiveness. Compared with an AZA-based regimen, MMF had an incremental cost of $\$ 5,976$ and gain of 0.9260 QALY, with an ICER of $\$ 6,454$ per QALY (Table 4(a)). (ii) Probabilistic Sensitivity Analyses. The CEAC showed that an MMF-based regimen had a near $100 \%$ probability of being cost-effective over a 40-year time frame, at WTP thresholds of \$50,000 and \$100,000/QALY (Supplemental Figure 5).

(iii) Sensitivity Analyses. As shown in Table 4(b), the ICER (MMF versus AZA) decreased over time such that MMF became cost-effective compared to AZA at 10 years postmaintenance therapy (Figures 3(b) and 3(c)). We also conducted sensitivity analyses by varying the probability of ESRD in the relapse state, demonstrating that the higher the risk of ESRD, the greater the cost-effectiveness of MMF versus AZA. Given the higher baseline risk of ESRD on AZA maintenance therapy, any incremental increase in this risk would disproportionately affect AZA (higher costs and lower QALY) as compared to MMF, resulting in a lower ICER (MMF versus AZA). We conducted other sensitivity analyses by excluding indirect costs, varying utility weights or discount rates, with the ICER (MMF versus AZA) well below the WTP \$50,000/QALY threshold (Table 4(b)).

(iv) Scenario Analysis. The ICER of the base-case $(\$ 6,454 / \mathrm{QALY})$ was based on the assumption that the treatment effect of MMF and AZA during the trial phase would persist over a lifetime. As shown in Table 4(b), MMF remained cost-effective over lifetime even if the treatment effect of both therapies diminished by $1 \%$ or $2 \%$ per year. However, assuming no treatment benefit after 3 years of maintenance therapy with either agent, MMF was not cost-effective compared to AZA (\$428,894/QALY).

3.3.2. Subgroup Analysis. MMF had favorable ICER compared to AZA over lifetime using ALMS (\$4,394/QALY) and MAINTAIN data (\$54,891/QALY), below the WTP $\$ 50,000-$ $\$ 100,000 /$ QALY (Table 4(a)).

\section{Discussion}

MMF and AZA are the most widely used therapeutic agents for long-term maintenance therapy of proliferative LN [35]. However, there is no consensus on the agent of choice, reflected by current clinical practice guideline recommendations $[3,4]$. To evaluate the cost-effectiveness of MMF versus AZA-based regimens, we developed a Markov model to simulate patient-oriented outcomes, both from short-term and from lifetime horizon.

We found poor cost-effectiveness of MMF versus AZAbased therapy at 3 years, with an ICER \$2,630,592/QALY. The ICER of MMF versus AZA remained substantially elevated in sensitivity analyses, even in conditions biased against AZA. Over a lifetime, however, our base-case analysis demonstrated MMF to be cost-effective compared to AZA, with an ICER \$6,454/QALY. Overall, the ICER results from various sensitivity analyses did not alter the conclusions of the lifetime model, except in an unlikely scenario where the treatment effect was nil after 3 years of maintenance therapy. In contrast to the initial 3-year time period, subgroup analysis of ALMS and MAINTAIN trials showed that the MMF-based 


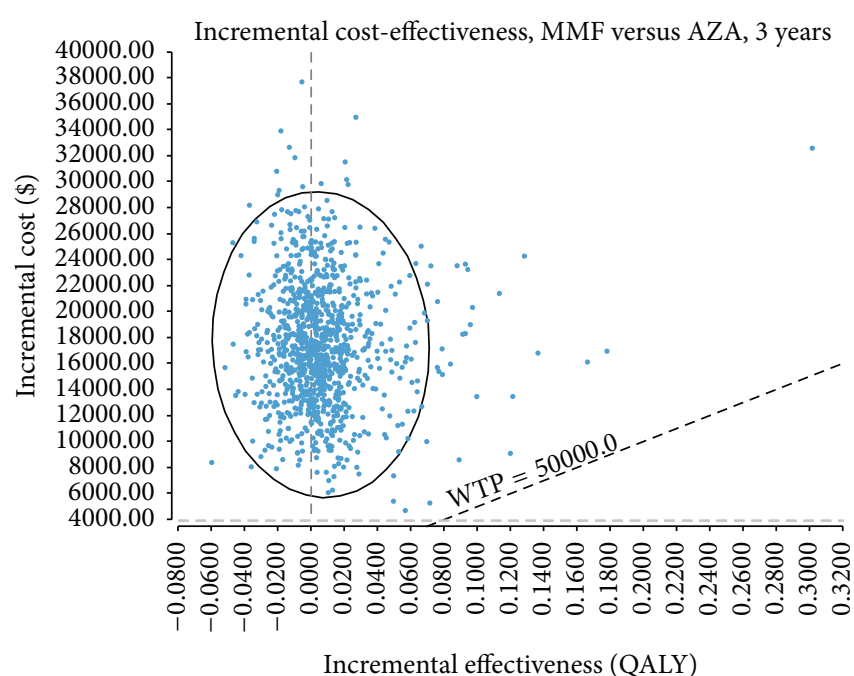

(a)

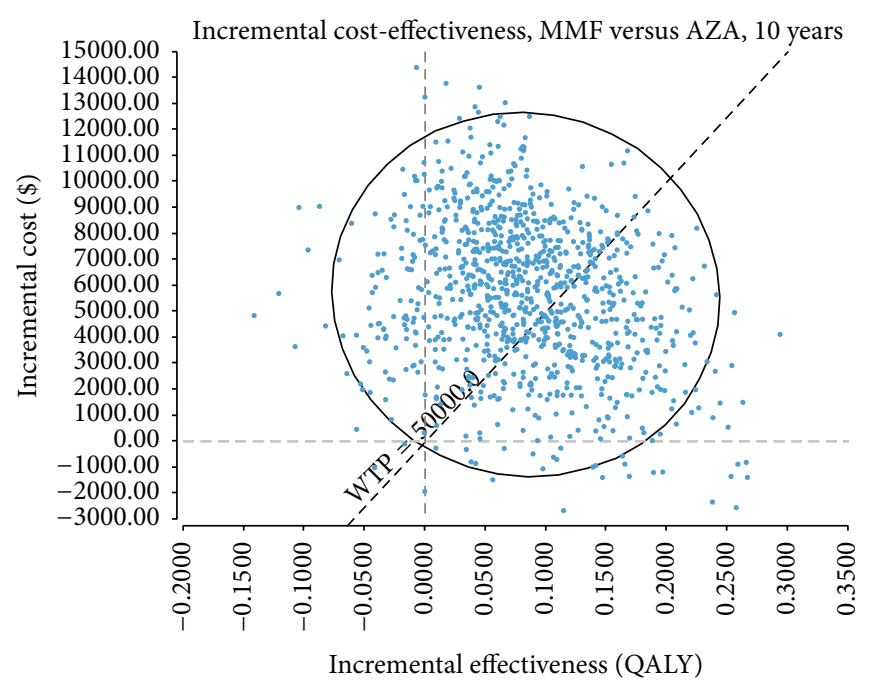

(b)

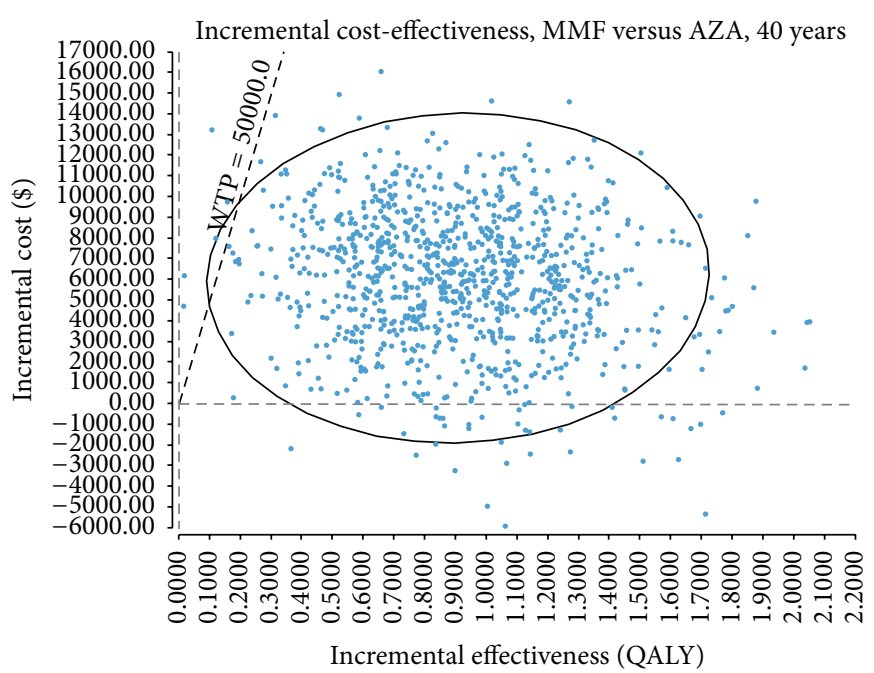

(c)

FIGURE 3: Incremental cost-effectiveness scatter plots of the base-case model. (a) 3 years; (b) 10 years after completing 3-year maintenance therapy; (c) 40 years after completing 3-year maintenance therapy. Each single point represents pairs of incremental cost and effectiveness values from probabilistic sensitivity analyses via second-order Monte Carlo simulation of 1,000 iterations. The ellipsis represents the 95\% confidence interval. The dotted diagonal line represents the WTP threshold of \$50,000/QALY. AZA: azathioprine; MMF: mycophenolate mofetil; WTP: willingness-to-pay; QALY: quality-adjusted life-years.

strategy was cost-effective compared to AZA from a lifetime perspective, at WTP $\$ 50,000-\$ 100,000 /$ QALY.

To our knowledge, there are only two published costeffectiveness analyses of LN treatment. Wilson et al. estimated the cost-utility of MMF versus IV CYC as induction therapy for 6 months from the perspective of the National Health Service in the United Kingdom (UK) [36]. Their analysis suggested that MMF was likely to result in better quality of life and be less expensive than IV CYC as induction therapy. More recently, Mohara et al. conducted a lifetime costutility analysis of four different immunosuppressive regimens for LN patients in Thailand [37]. The study demonstrated that, from a Thai perspective, induction with IV CYC followed by AZA was the most cost-effective regimen of all the alternatives. Our study reached different conclusions due to notable mutual differences in the model structure and assumptions, setting (US versus Thailand), target population, cost and utility parameters, and transition probabilities; and the Cochrane meta-analysis was not used as data source in Mohara's study.

Our study has certain limitations. First, lifetime modeling required extrapolation of data beyond the period observed in clinical trials which could lead to inconsistent results. We therefore assessed the uncertainty of future treatment benefit by conducting sensitivity analyses based on established guidelines [38]. Second, we assume that patients in our model receive immunosuppressive maintenance therapy for 3 years based on published clinical trials [13]. Due to lack of data from 
TABLE 4: (a) Costs, effectiveness, and incremental cost effectiveness ratios (ICER) of the base-case and individual clinical trials in lifetime model (40 years). (b) Sensitivity analysis of lifetime model using Cochrane data.

(a)

\begin{tabular}{|c|c|c|c|c|c|}
\hline Scenarios & Total cost $(\$)$ & $\begin{array}{c}\text { Total } \\
\text { effectiveness } \\
\text { (QALY) }\end{array}$ & $\begin{array}{c}\text { Incremental } \\
\text { costs }(\$)\end{array}$ & $\begin{array}{c}\text { Incremental } \\
\text { effectiveness } \\
(\text { QALY) }\end{array}$ & $\begin{array}{c}\text { ICER } \\
(\$ / Q A L Y)\end{array}$ \\
\hline \multicolumn{6}{|c|}{ Cochrane (base-case) } \\
\hline AZA & $478,333.42$ & 14.1623 & & & \\
\hline MMF & $484,309.78$ & 15.0882 & 5976.36 & 0.9260 & 6454.24 \\
\hline \multicolumn{6}{|l|}{ Subgroups } \\
\hline \multicolumn{6}{|l|}{ ALMS } \\
\hline AZA & $485,791.18$ & 13.5979 & & & \\
\hline MMF & $493,953.07$ & 15.4554 & 8161.89 & 1.8575 & 4393.90 \\
\hline \multicolumn{6}{|c|}{ MAINTAIN } \\
\hline AZA & $469,825.11$ & 14.0140 & & & \\
\hline MMF & $486,758.11$ & 14.3225 & $16,933.00$ & 0.3085 & $54,891.42$ \\
\hline
\end{tabular}

QALY: quality-adjusted life-years; AZA: azathioprine; MMF: mycophenolate mofetil.

(b)

\begin{tabular}{|c|c|}
\hline Scenarios & ICER MMF versus AZA (US\$) \\
\hline Base-case (40-year time horizon) & $\$ 6,454.24$ \\
\hline Excluding indirect costs & Dominant $^{\mathrm{a}}$ \\
\hline \multicolumn{2}{|l|}{ Utility } \\
\hline Remission 0.8 (versus base-case 0.7 ) & $\$ 4067.55$ \\
\hline Relapse 0.5 (versus base-case 0.6 ) & $\$ 5,808.27$ \\
\hline Relapse 0.7 (versus base-case 0.6 ) & $\$ 7,695.58$ \\
\hline \multicolumn{2}{|l|}{ Increase in probability of ESRD with relapse } \\
\hline $0.5 \%$ per year & $\$ 4590.37$ \\
\hline $1.0 \%$ per year & $\$ 3112.96$ \\
\hline $2.0 \%$ per year & $\$ 2717.08$ \\
\hline \multicolumn{2}{|l|}{ Extrapolated treatment effect after 3-year maintenance therapy } \\
\hline Same as during treatment phase (base-case) & $\$ 6,454.24$ \\
\hline No treatment effect from both MMF and AZA during extrapolated phase ${ }^{\mathrm{b}}$ & $\$ 428,894.16$ \\
\hline Treatment effect from both MMF and AZA decreases $1 \%$ per year ${ }^{c}$ & $\$ 15,096.38$ \\
\hline Treatment effect from both MMF and AZA decreases $2 \%$ per year $^{c}$ & $\$ 25,713.36$ \\
\hline \multicolumn{2}{|l|}{ Time horizon (number of years after maintenance therapy) } \\
\hline 5 years & $\$ 513,712.88$ \\
\hline 10 years & $\$ 67,203.94$ \\
\hline 20 years & Dominant $^{\mathrm{a}}$ \\
\hline 30 years & $\$ 5,232.11$ \\
\hline \multicolumn{2}{|l|}{ Discount rate (base-case 3\% for costs and utility) } \\
\hline $0 \%$ & $\$ 5,830.11$ \\
\hline $5 \%$ & $\$ 10,230.91$ \\
\hline $7 \%$ & $\$ 14,374.62$ \\
\hline
\end{tabular}

ICER: incremental cost effectiveness ratio; AZA: azathioprine; MMF: mycophenolate mofetil; ESRD: end stage renal disease.

${ }^{a} \mathrm{MMF}$ is less costly and more effective than AZA-based regimen.

${ }^{\mathrm{b}}$ Assuming $100 \%$ probability of relapse during remission on either MMF or AZA after completing 3-year maintenance therapy.

${ }^{\mathrm{c}}$ Assuming $1 \%$ or $2 \%$ per year increase in relapse during remission on either MMF or AZA after completing 3-year maintenance therapy.

long-term randomized studies of maintenance therapy in patients with proliferative lupus nephritis, we did not model scenarios whereby patients are kept on maintenance therapy for $>3$ years. Modeling such scenarios based on incomplete medical evidence would compromise face validity. Furthermore, we would not be able to test the model for external validity (comparing predicted results from the model with actual event data) [39]. Third, the total costs of each strategy 
are likely underestimated since cost data are based on the Tri-Nation Study which included lupus patients from the US, Canada, and UK $[18,19]$. This study estimated that direct and indirect costs in the US are 20\% and 29\% higher, respectively, than Canada. However, this underestimation of total costs does not change the conclusions of our analysis which is based on incremental calculations. Fourth, our model included utility scores that were measured by VAS [17, 23] which does not involve a trade-off that a subject must choose between the health states, in contrast to the standard gamble and time trade-off techniques. However, VAS was demonstrated to be a valid and reliable measure of health related quality of life in a SLE cohort [40]. Lastly, we incorporated major infection in the model as the most severe side effect of immunosuppressive therapy but did not consider gastrointestinal disturbance, leukopenia, alopecia, or infertility.

Acknowledging these limitations, our study does suggest that, from a cost-effectiveness standpoint, an AZAbased regimen confers greater value than MMF for the maintenance therapy of proliferative LN in the short term. Value of information analysis suggests a population EVPI of \$2,058,206 at WTP \$100,000/QALY which represents the expected maximum gain on investment on further research. The implication is that spending more than this amount on additional data collection would represent a poor investment of limited research funds. In contrast to the short-term perspective, MMF is cost-effective compared to AZA at the standard WTP threshold in the US over the patients' lifetime. Despite the relatively higher upfront costs of MMF during the 3-year maintenance phase, its salutary effects (lower risk of LN relapse and progression to ESRD compared to AZA) make MMF a cost-effective option over the long term. Our analysis is consistent with the general notion that the time frame of a model should be sufficiently long to capture future differences in costs and health outcomes between treatment strategies.

Given the substantial economic burden of LN in our healthcare system, the findings of this study should be an important factor in selecting the optimal maintenance regimen for patients with proliferative LN. Furthermore, these findings may provide useful information to support more individualized therapy.

\section{Disclaimer}

The views expressed in this paper are those of the authors and do not necessarily reflect the official policy or position of the Department of the Army, the Department of the Navy, the Department of Defense, or the United States government.

\section{Conflict of Interests}

The authors report no conflict of interests.

\section{Acknowledgment}

The authors would like to acknowledge Annie Nguyen, PharmD, for her assistance in obtaining data for drug costs.

\section{References}

[1] K. A. Slawsky, A. W. Fernandes, L. Fusfeld, S. Manzi, and T. F. Goss, "A structured literature review of the direct costs of adult systemic lupus erythematosus in the US," Arthritis Care \& Research, vol. 63, no. 9, pp. 1224-1232, 2011.

[2] G. Carls, T. Li, P. Panopalis et al., "Direct and indirect costs to employers of patients with systemic lupus erythematosus with and without nephritis," Journal of Occupational and Environmental Medicine, vol. 51, no. 1, pp. 66-79, 2009.

[3] Kidney Disease: Improving Global Outcomes (KDIGO) Glomerulonephritis Work Group, "KDIGO clinical practice guideline for glomerulonephritis," Kidney International Supplements, vol. 2, no. 2, pp. 139-274, 2012.

[4] B. H. Hahn, M. A. McMahon, A. Wilkinson et al., "American College of Rheumatology guidelines for screening, treatment, and management of lupus nephritis," Arthritis Care and Research, vol. 64, no. 6, pp. 797-808, 2012.

[5] F. A. Houssiau, D. D’Cruz, S. Sangle et al., "Azathioprine versus mycophenolate mofetil for long-term immunosuppression in lupus nephritis: results from the MAINTAIN Nephritis Trial," Annals of the Rheumatic Diseases, vol. 69, no. 12, pp. 2083-2089, 2010.

[6] M. A. Dooley, D. Jayne, E. M. Ginzler et al., "Mycophenolate versus azathioprine as maintenance therapy for lupus nephritis," The New England Journal of Medicine, vol. 365, no. 20, pp. 18861895, 2011.

[7] A. M. Gray, P. M. Clarke, J. L. Wolstenholme, and S. Wordsworth, Applied Methods of Cost-Effectiveness Analysis in Health Care, Oxford University Press, New York, NY, USA, 2011.

[8] D. Husereau, M. Drummond, S. Petrou et al., "Consolidated health economic evaluation reporting standards (CHEERS) explanation and elaboration: a report of the ISPOR health economic evaluation publication guidelines good reporting practices task force," Value in Health, vol. 16, no. 2, pp. 231-250, 2013.

[9] B. Sprangers, M. Monahan, and G. B. Appel, "Diagnosis and treatment of lupus nephritis flares-an update," Nature Reviews Nephrology, vol. 8, no. 12, pp. 709-717, 2012.

[10] R. J. Falk, P. H. Schur, and G. B. Appel, "Therapy of resistant or relapsing diffuse or focal proliferative lupus nephritis," UpToDate, May 2013, http://www.uptodate.com/.

[11] Bureau of Labor Statistics, "Consumer Price Index," December 2013, http://www.bls.gov/cpi/home.htm.

[12] Red Book Online, June 2013, http://www.redbook.com/redbook/online/.

[13] L. Henderson, P. Masson, J. C. Craig et al., "Treatment for lupus nephritis," Cochrane Database of Systematic Reviews, vol. 12, Article ID CD002922, 2012.

[14] K. H. Costenbader, A. Desai, G. S. Alarcón et al., “Trends in the incidence, demographics, and outcomes of end-stage renal disease due to lupus nephritis in the US from 1995 to 2006," Arthritis \& Rheumatism, vol. 63, no. 6, pp. 1681-1688, 2011.

[15] F. A. Sonnenberg and J. R. Beck, "Markov models in medical decision making: a practical guide," Medical Decision Making, vol. 13, no. 4, pp. 322-338, 1993.

[16] Centers for Medicare \& Medicaid Services, December 2013, http://www.cms.gov/Medicare/Medicare-Fee-for-Service-Payment/HospitalOutpatientPPS/Addendum-A-and-AddendumB-Updates.html. 
[17] A. E. Clarke, P. Panopalis, M. Petri et al., "SLE patients with renal damage incur higher health care costs," Rheumatology, vol. 47, no. 3, pp. 329-333, 2008.

[18] A. E. Clarke, M. Petri, S. Manzi et al., "The systemic lupus erythematosus tri-nation study: absence of a link between health resource use and health outcome," Rheumatology, vol. 43, no. 8, pp. 1016-1024, 2004.

[19] P. Panopalis, M. Petri, S. Manzi et al., "The systemic lupus erythematosus tri-nation study: cumulative indirect costs," Arthritis Care and Research, vol. 57, no. 1, pp. 64-70, 2007.

[20] US Renal Data System, USRDS 2012 Annual Data Report: Atlas of Chronic Kidney Disease and End-Stage Renal Disease in the United States, National Institutes of Health, National Institute of Diabetes and Digestive and Kidney Diseases, Bethesda, Md, USA, 2012.

[21] Healthcare Cost and Utilization Project and Agency for Healthcare Research and Quality, 2013, http://hcupnet.ahrq.gov/ HCUPnet.jsp?Id=5452BD185046B6A8\&Form=DispTab\&JS= Y\&Action=\%3E\%3ENext\%3E\%3E\&GoTo=MAINSEL.

[22] Y. S. Liem, J. L. Bosch, and M. G. M. Hunink, "Preferencebased quality of life of patients on renal replacement therapy: a systematic review and meta-analysis," Value in Health, vol. 11, no. 4, pp. 733-741, 2008.

[23] C. Grootscholten, F. J. Snoek, M. Bijl et al., "Health-related quality of life and treatment burden in patients with proliferative lupus nephritis treated with cyclophosphamide or azathioprine/ methylprednisolone in a randomized controlled trial," Journal of Rheumatology, vol. 34, no. 8, pp. 1699-1707, 2007.

[24] K. C. Tse, C. S. O. Tang, W. I. Lio, M. F. Lam, and T. M. Chan, "Quality of life comparison between corticosteroid-andmycofenolate mofetil and corticosteroid-and-oral cyclophosphamide in the treatment of severe lupus nephritis," Lupus, vol. 15 , no. 6, pp. 371-379, 2006.

[25] Tufts' Cost-Effectiveness Analysis Registry, December 2013, https://research.tufts-nemc.org/cear4/Home.aspx.

[26] M. F. Drummond, M. J. Sculpher, G. W. Torrance, B. J. O’Brien, and G. L. Stoddart, Methods for the Economic Evaluation of Health Care Programmes, Oxford University Press, New York, NY, USA, 3rd edition, 2005.

[27] S. Bernatsky, J.-F. Boivin, L. Joseph et al., "Mortality in systemic lupus erythematosus," Arthritis \& Rheumatism, vol. 54, no. 8, pp. 2550-2557, 2006.

[28] E. Arias, "United States life tables, 2007," National Vital Statistics Reports, vol. 59, pp. 1-60, 2011.

[29] S. Sule, B. Fivush, A. Neu, and S. Furth, "Increased risk of death in pediatric and adult patients with ESRD secondary to lupus," Pediatric Nephrology, vol. 26, no. 1, pp. 93-98, 2011.

[30] R. S. Braithwaite, D. O. Meltzer, J. T. King Jr., D. Leslie, and M. S. Roberts, "What does the value of modern medicine say about the $\$ 50,000$ per quality-adjusted life-year decision rule?" Medical Care, vol. 46, no. 4, pp. 349-356, 2008.

[31] M. C. Weinstein, J. E. Siegel, M. R. Gold, M. S. Kamlet, and L. B. Russell, "Recommendations of the panel on cost-effectiveness in health and medicine," Journal of the American Medical Association, vol. 276, no. 15, pp. 1253-1258, 1996.

[32] B. Groot Koerkamp, M. C. Weinstein, T. Stijnen, M. H. Heijenbrok-Kal, and M. G. M. Hunink, "Uncertainty and patient heterogeneity in medical decision models," Medical Decision Making, vol. 30, no. 2, pp. 194-205, 2010.
[33] F. A. Houssiau, C. Vasconcelos, D. D’Cruz et al., “The 10year follow-up data of the Euro-Lupus Nephritis Trial comparing low-dose and high-dose intravenous cyclophosphamide," Annals of the Rheumatic Diseases, vol. 69, no. 1, pp. 61-64, 2010.

[34] C. C. Mok, R. C. L. Kwok, and P. S. F. Yip, "Effect of renal disease on the standardized mortality ratio and life expectancy of patients with systemic lupus erythematosus," Arthritis and Rheumatism, vol. 65, no. 8, pp. 2154-2160, 2013.

[35] O. Lenz, A. A. Waheed, A. Baig, A. Pop, and G. Contreras, "Lupus nephritis: maintenance therapy for lupus nephritis-do we now have a plan?" Clinical Journal of the American Society of Nephrology, vol. 8, no. 1, pp. 162-171, 2013.

[36] E. C. F. Wilson, D. R. W. Jayne, E. Dellow, and R. J. Fordham, "The cost-effectiveness of mycophenolate mofetil as firstline therapy in active lupus nephritis," Rheumatology, vol. 46, no. 7, pp. 1096-1101, 2007.

[37] A. Mohara, R. P. Velasco, N. Praditsitthikorn, Y. Avihingsanon, and Y. Teerawattananon, "A cost-utility analysis of alternative drug regimens for newly diagnosed severe lupus nephritis patients in Thailand," Rheumatology, vol. 53, no. 1, Article ID ket304, pp. 138-144, 2014.

[38] NICE guide to the methods of technology appraisal 2013, 2014, http://www.nice.org.uk/article/PMG9/chapter/5-The-referencecase.

[39] D. M. Eddy, W. Hollingworth, J. J. Caro, J. Tsevat, K. M. McDonald, and J. B. Wong, "Model transparency and validation: a report of the ISPOR-SMDM modeling good research practices task force-7," Medical Decision Making, vol. 32, no. 5, pp. 733743, 2012.

[40] A. D. Moore, A. E. Clarke, D. S. Danoff et al., "Can health utility measures be used in lupus research? A comparative validation and reliability study of 4 utility indices," Journal of Rheumatology, vol. 26, no. 6, pp. 1285-1290, 1999. 


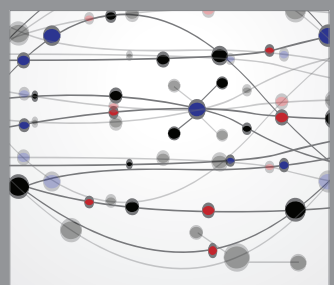

The Scientific World Journal
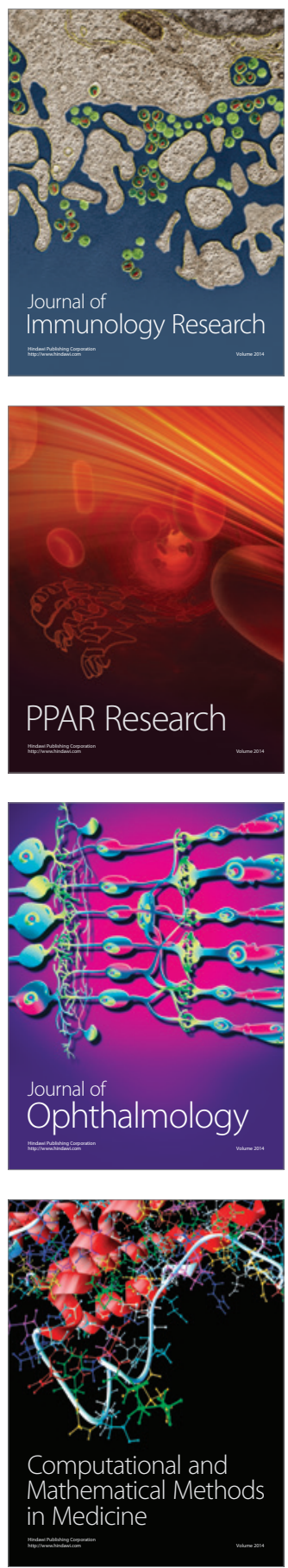

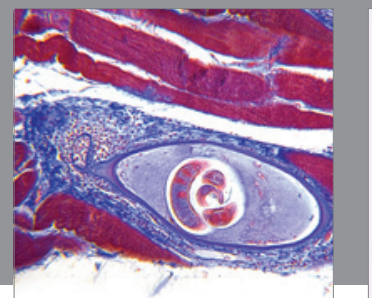

Gastroenterology

Research and Practice
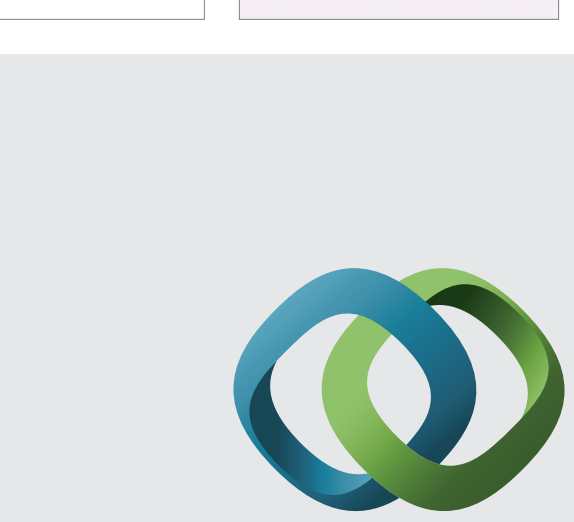

\section{Hindawi}

Submit your manuscripts at

http://www.hindawi.com
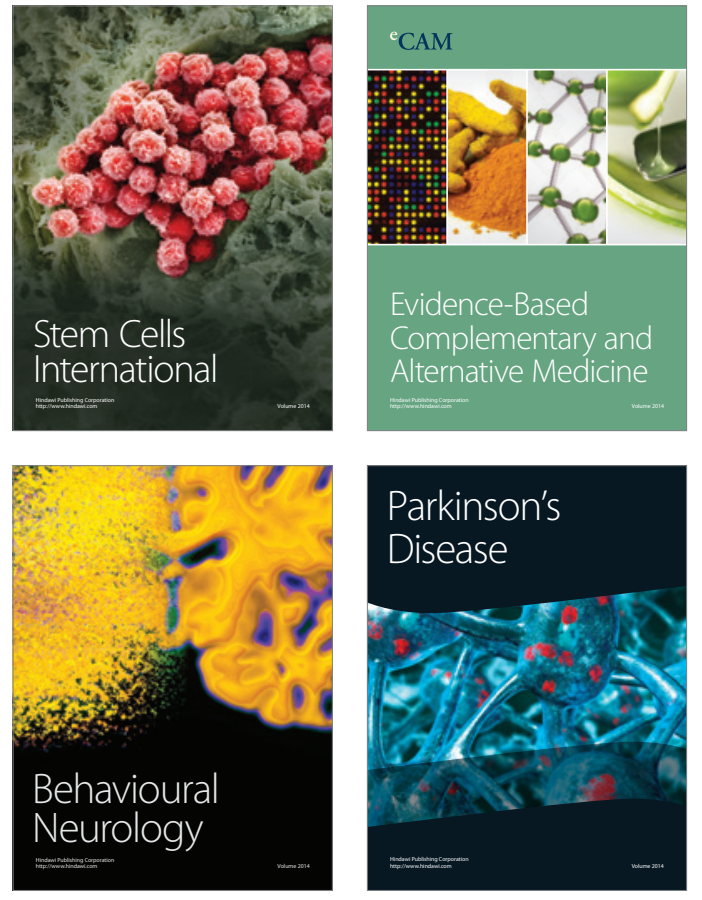
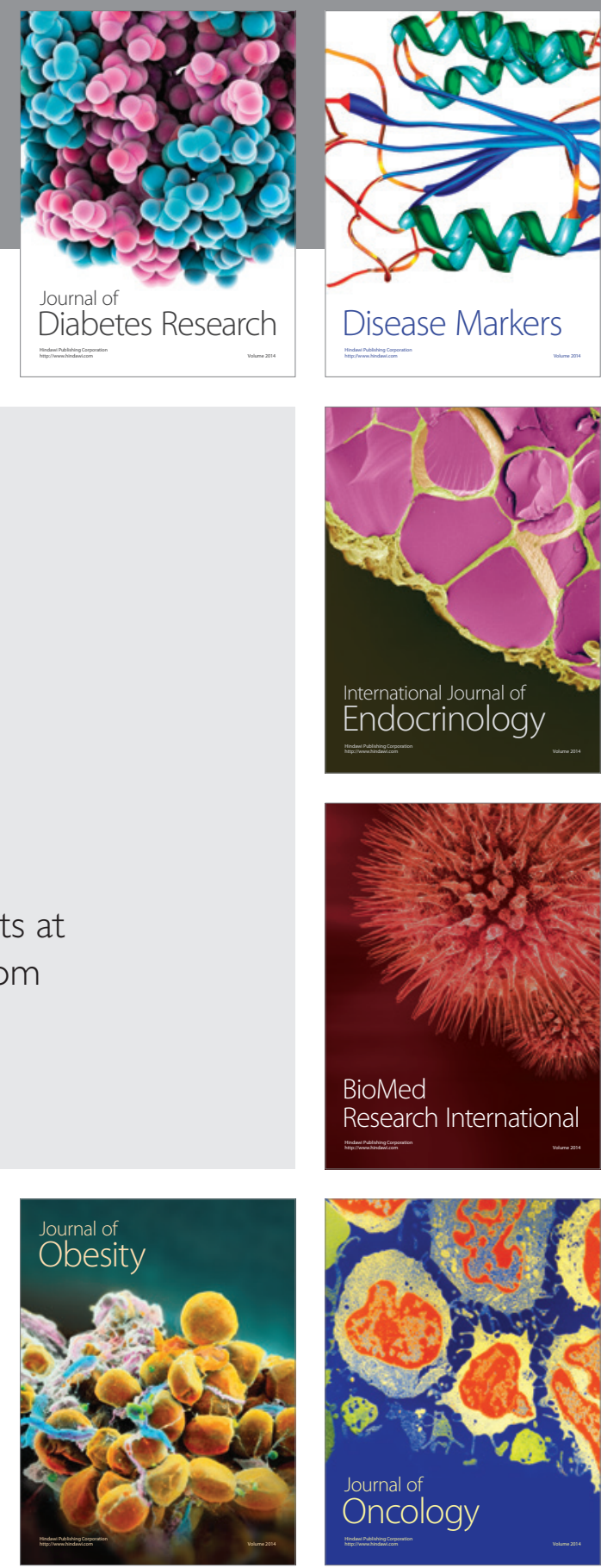

Disease Markers
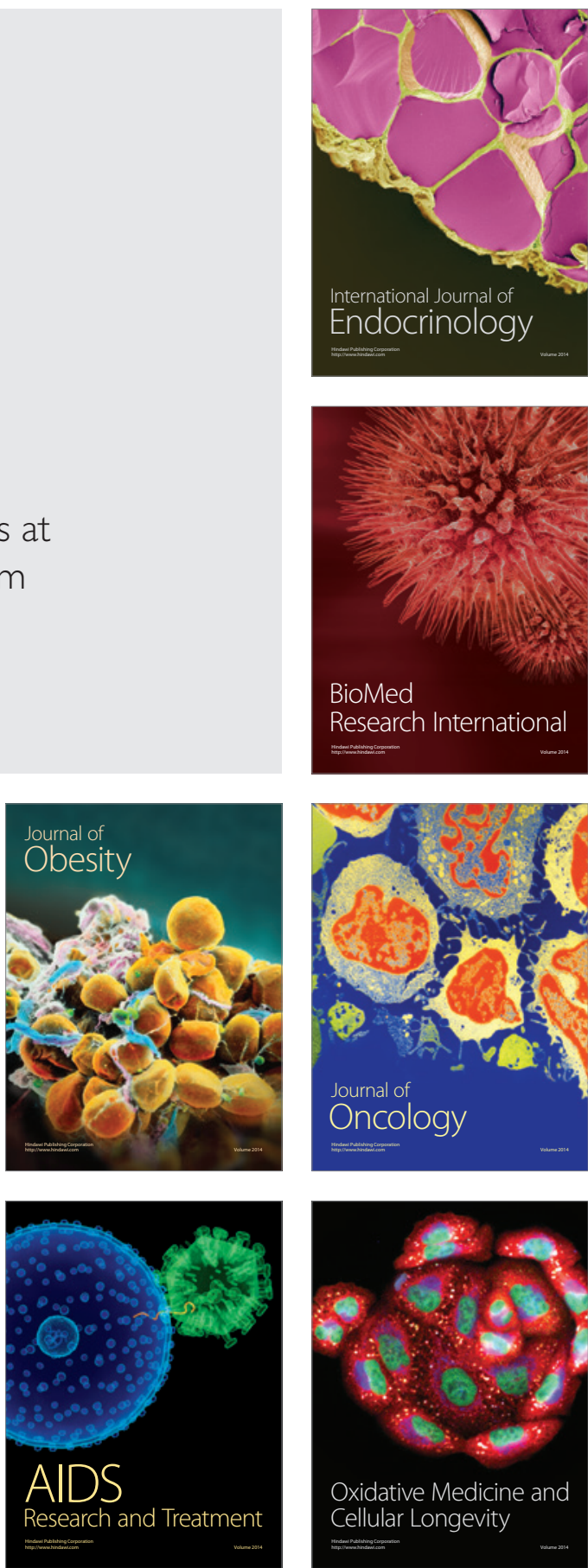